\title{
Use of clinical and service indicators to decrease the treatment gap for mental disorders: a global perspective
}

\author{
SHEKHAR SAXENA \\ Department of Mental Health and Substance Abuse, World Health Organization, Geneva (Switzerland)
}

Against the stark reality of the fact that worldwide most people with mental illness are not treated (Thornicroft, 2007), the impact of any serious undertaking in the area of global mental health has to be judged against the benchmark of whether it leads to more people being treated. Treatment gap for mental disorders can be defined as the proportion of individuals who require but do not receive treatment. Some recent studies have demonstrated that treatment gap for mental disorders is surprising high. A review of international literature (Kohn et al., 2004) revealed that one-third of all individuals with Schizophrenia and other non-affective psychoses do not receive any treatment. Figures for depression, dysthymia, bipolar disorder, panic, obsessive compulsive and anxiety disorders are all around 50\%. The World Mental Health Survey data show that 35 to $50 \%$ of serious cases in developed countries and 76 to $85 \%$ in developing countries received no treatment in the previous year (WHO World Mental Health Consortium, 2004). This commentary examines a few selected issues around the role and limitations of clinical and service indicators in decreasing the treatment gap in a global perspective. It also introduces WHO's indicator scheme for assessing mental health systems.

\section{ARE INDICATORS NEEDED IN LOW RESOURCE SETTINGS?}

The primary reasons for the large treatment gap for mental disorders seem to be scarcity, inequity and inefficiency of resources for mental health (Saxena et al.,

Address for correspondence: Dr. S. Saxena, Department of Mental Health and Substance Abuse World Health Organization CH-1211, Geneva (Switzerland).

E-mail: saxenas@who.int

Declaration of Interest: None. 2007b). The budgetary allocation for mental health from the total health budget is usually very small; the median percentage for this for all countries is 3.76 and for low income countries, 2.26. A large number of countries spend less than $1 \%$ of their health budget on mental health. The availability of human resources is also highly inadequate. The median numbers of psychiatrists and psychiatric nurses in low income countries are 0.05 and 0.16 per 100,000 population- hardly adequate to provide even basic mental health care to all who need it. In view of this severe lack of financial and human resources for mental health care, are clinical and service indicators needed for low and middle income countries? If yes, what should the indicators look like?

If evidence based clinical interventions and services are necessary for resource rich settings, these are even more needed for resource poor settings. When resources are scarce, none should be wasted. Indicators serve the extremely useful function of facilitating evaluation of current practices, identification of strengths and weaknesses and making possible comparisons across services and time. However, information collected with indicators also makes corrective action possible, not only at services level but also at the level of policy and programme. Resources can be enhanced, distributed more equitably and utilized more efficiently based on service and clinical indicators. For all these reasons, the SIEP-DIRECT'S Project (DIscrepancy between $\mathbf{R}$ outine practice and Evidence in psychiatric Community Treatments on Schizophrenia) (Ruggeri et al., 2008; Semisa et al., 2008; Ruggeri et al., 2008 ) is a valuable and innovative initiative and provides useful tools to optimize resource allocation and use.

\section{WHICH INDICATORS WOULD BE MOST USEFUL IN LOW RESOURCE SETTINGS?}

Though indicators serve very useful needs globally, it is important to match the level and specificity of indica- 
tors to their expected use. High level aggregate service indicators are more useful for policy, plans and service organization. More specific fine grained indicators are likely to be needed for improving the fidelity of delivery of interventions and for training of clinical care providers. At different stage of service development and resource availability, the mix of indicators will vary. In general, service systems that are poorly resourced, tend to benefit more from aggregate high level service and system level indicators. However, the current availability of indicators seems not to be sensitive to this need (Saxena et al., 2006). Evidence for clinical guidelines and indicators focusing on discrete interventions is accumulating at a rapid pace. However, evidence for effectiveness and costeffectiveness of services is lagging behind. For example, National Schizophrenia Guidelines for UK (National Institute for Mental Health, 2003) concluded that evidence for service level interventions for schizophrenia is largely insufficient to make definite recommendations. This creates the situation in which the confidence with which specific services for mental disorders can be recommended is much lower than the confidence for specific interventions. Scientific evidence seems to provide less help precisely in the area that is the main priority for low resource settings.

\section{SHOULD INDICATORS COVER HUMAN RIGHTS ASPECTS?}

Abuse or neglect of basic human rights of people with mental disorders are quite commonly encountered in mental health services (World Health Organization, 2005b). Clinical and service indicators need to cover the human rights aspects of care along with the interventions being delivered. Human rights guidance needs to be a reflection of values rather than of the evidence for effectiveness. Unfortunately, currently available clinical guidelines all but ignore human rights issues. This results in an erroneous message to service providers and trainees that they are accountable for the delivery of scientific evidence based interventions to their patients while human rights and other value based aspects are peripheral to their responsibilities. This situation must change and one of the ways to bring this about is to integrate human rights issues in clinical and service guidelines and indicators.

\section{WHO-AIMS}

In view of the serious and urgent need to have reliable and accurate information on mental health systems and services from low and middle income countries, World Health Organization has developed WHO-AIMS (The World Health Organization Assessment Instrument for Mental Health Systems). This instrument is an indicator scheme that systematically assesses important attributes of mental health systems and services. Details of the rationale and the process followed to develop this instrument are described elsewhere (World Health Organization, 2005a; Saxena et al., 2007a). The instrument contains 155 measures covering six core domains: the policy and legislative framework for mental health, mental health services, mental health in primary health care, human resources, links with other sectors, and monitoring and research. In terms of the overall structure and focus of the instrument, WHO-AIMS contains mainly process and structure measures rather than outcome measures. The primary reason is that the majority of low-income countries still lack the basic mental health infrastructure, such as community-based services, or a functioning information system. Thus, collecting data on clinical interventions or practices as well as on outcomes would be extremely difficult, if not impossible within reasonable effort. WHO-AIMS includes indicators for not only overall national/provincial systems but also concrete service indicators like availability of human, financial and other resources and the throughput and characteristics of patients being cared for. A number of indicators are devoted to human rights considerations (e.g. inspection of human rights in mental health facilities, physical restraints and seclusion in mental health facilities).WHO-AIMS is currently being used in more than 60 low and middle income countries and final reports from more that 40 countries are already available (http://www.who.int/mental_health/evidence/ WHO-AIMS/en/index.html). A number of these countries are using the WHO-AIMS data and reports for strengthening their mental health systems.

Since scaling-up of services seems the highest priority for most low and middle income countries (Lancet Global Mental Health Group, 2007), clinical and service guidelines need to be developed in a way that they serve the need for enhancing the coverage with services rather than to focus exclusively on improving the quality of services. In some cases, these two objectives will be mutually incompatible; in resource poor settings, a few people can be given excellent care or many more people can be given basic (but still effective) care. Guidelines and indicators need to be sensitive to these possibly contradictory needs and strike a good balance. 


\section{REFERENCES}

Kohn R., Saxena D., Levav I. \& Saraceno B. (2000). The treatment gap in mental health care. Bulletin of the World Health Organization 8, $858-864$.

Lancet Global Mental Health Group (2007). Scale up services for mental disorders: a call for action. Lancet 370, 1241-1252.

National Institute for Mental Health (2003). Schizophrenia: Full National Clinical Guidelines on Core Interventions in Primary and Secondary Care. Gaskell and British Psychological Society: London.

Ruggeri M. (2008). Guidelines for treating mental illness: love them, hate them. Can the SIEP-DIRECT'S Project serve in the search for a happy medium? Epidemiologia e Psichiatria Sociale 17(4), 270-277.

Ruggeri M., Lora A. \& Semisa D. on behalf of the SIEP-DIRECT'S Group (2008). The SIEP-DIRECT'S Project on the discrepancy between routine practice and evidence. An outline of the main findings and the practical implications for the future of community based mental health services. Epidemiologia e Psichiatria Sociale 17(4), 358-367.

Saxena S., van Ommeren M., Lora A. \& Saraceno B. (2006). Monitoring of mental health systems and services - comparison of four existing indicator schemes. Social Psychiatry and Psychiatric Epidemiology 41, 488-497.

Saxena S., Lora A., van Ommeren M., Barrett T., Morris J. \& Saraceno B. (2007a). WHO's assessment instrument for mental health sys tems: Collecting essential information for policy and service delivery. Psychiatric Services 58(6), 816-821.

Saxena S., Thornicroft G., Knapp M. \& Whiteford H. (2007b). Resources for mental health: scarcity, inequity, and inefficiency. Lancet 370, 878-889.

Semisa D., Lora A., Morosini P. \& Ruggeri M. (2008a). The SIEPDIRECT'S Project on the discrepancy between routine practice and evidence in the treatment of schizophrenia. The design, the indicators, and the methodology of the study (in Italian). Epidemiologia $e$ Psichiatria Sociale 17(4), 278-290.

Thornicroft G. (2007). Most people with mental illness are not treated. Lancet 370, 807-808.

WHO World Mental Health Consortium (2004). Prevalence, severity and unmet need for treatment of mental disorders in the World Mental Health Organization World Mental Health Surveys. Journal of the American Medical Association 291, 2581-2590.

World Health Organization (2005a). World Health Organization Assessment Instrument for Mental Health Systems (WHO-AIMS) Version 2.2. World Health Organization: Geneva. Retrieved August 19, 2008, from http://www.who.int/mental_health/evidence/AIMS WHO_2_2.pdf

World Health Organization (2005b). Resource Book on Mental Health, Human Rights and Legislation. WHO: Geneva. 